\title{
Dual Schur Complement Method for Semi-Definite Problems
}

\author{
Daniel J. Rixen
}

\section{Introduction}

Semi-definite problems are encountered in a wide variety of engineering problems. Most domain decomposition methods efficient for parallel computing are based on iterative schemes and rarely address the problem of checking the problem's singularity and computing the null space. In this paper we present a simple and efficient method for checking the singularity of an operator and for computing a null space when solving an elliptic structural problem with a dual Schur complement approach.

The engineering community has long been reluctant to use iterative solvers mainly because of their lack of robustness. With the advent of parallel computers, domain decomposition methods received a lot of attention which resulted in some efficient, scalable and robust solvers $[\mathbf{3}, \mathbf{6}]$. The Finite Element Tearing and Interconnecting method (FETI) has emerged as one of the most useful techniques and is making its way in structural and thermal commercial softwares $[\mathbf{1}, \mathbf{4}]$.

So far, the issue of semi-definite problems in FETI has not been fully addressed although a broad range of engineering problems are singular. For instance, the static and vibration analysis of satellites, aircrafts or multi-body structures is governed by $[5]$

$$
A x=b
$$

where $A$ is a symmetric semi-definite positive stiffness matrix, $x$ are the structural displacements and $b$ is the vector of external forces. The zero energy modes $u_{i}$, $i=1, \ldots m$, define a null space such that

$$
A u_{i}=0 \quad i=1, \ldots m
$$

and a solution exists for problem (1) only if

$$
u_{i}^{T} b=0 \quad i=1, \ldots m
$$

When using direct solvers to solve a singular problem such as (1), the null space is obtained as a by-product of the factorization when detecting zero pivots [5]. Unfortunately, when iterative solvers are applied, the algorithms do not provide any

1991 Mathematics Subject Classification. Primary 65N55; Secondary 65F10, 15A03, 73C02. The author was supported by the Belgian National Science Foundation.

(C)1998 American Mathematical Society 
information on the singularity of the problem and the null space is never computed in the iteration process.

In this paper, a general Conjugate Gradient procedure for testing the singularity of an operator and for extracting the null space is presented. Iterative methods for computing a null space exist (e.g. singular value decomposition or inverse iteration with spectral shifting [5]), but they entail a tremendous computational cost when applied to large systems. Here we show how algorithms like Conjugate Gradient for solving linear systems can be adapted to check for singularity and to compute a null space, thereby adding only a small computational overhead and involving only minor alteration to the solution procedure. We will discuss its application to the FETI solver.

\section{Finite element tearing and inter-connecting}

2.1. The dual Schur complement formulation. The solution of a problem of the form (1) where $A$ is a symmetric positive matrix arising from the discretization of some second- or fourth-order elliptic structural mechanics problem on a domain $\Omega$, can be obtained by partitioning $\Omega$ into $N_{s}$ substructures $\Omega^{(s)}$, and gluing these with discrete Lagrange multipliers $\lambda[\mathbf{3}]$ :

$$
\begin{aligned}
A^{(s)} x^{(s)}+B^{(s)^{T}} \lambda & =b^{(s)} \quad s=1, \ldots, N_{s} \\
\sum_{s=1}^{s=N_{s}} B^{(s)} x^{(s)} & =0
\end{aligned}
$$

where the superscript $(s)$ denotes a quantity pertaining to $\Omega^{(s)}, B^{(s)}$ is a signed Boolean matrix such that $B^{(s)} x^{(s)}$ is the restriction of $x^{(s)}$ to the subdomain interface boundary and $\lambda$ are Lagrange multipliers associated to the interface compatibility constraints (5). From Eqs. (4), $x^{(s)}$ can be computed as

$$
x^{(s)}=A^{(s)^{+}}\left(b^{(s)}-B^{(s)^{T}} \lambda\right)+R^{(s)} \alpha^{(s)}
$$

where $A^{(s)^{+}}$denotes the inverse of $A^{(s)}$ if $\Omega^{(s)}$ is not singular, or a generalized inverse of $A^{(s)}$ otherwise. In the latter case, $R^{(s)}=\operatorname{Ker}\left(A^{(s)}\right)$ stores a basis of the null space of $A^{(s)}$ and is obtained during the factorization of $A^{(s)}$, and $\alpha^{(s)}$ stores the amplitudes of $R^{(s)}$. If $A^{(s)}$ is singular, (4) requires that

$$
R^{(s)^{T}}\left(b^{(s)}-B^{(s)^{T}} \lambda\right)=0
$$

From Eqs. (6) and (5), and recalling condition (7), the interface problem can be written as $[\mathbf{3}]$

$$
\left[\begin{array}{cc}
F_{I} & -G_{I} \\
-G_{I}^{T} & 0
\end{array}\right]\left[\begin{array}{l}
\lambda \\
\alpha
\end{array}\right]=\left[\begin{array}{c}
d \\
-e
\end{array}\right]
$$

where

$$
\begin{aligned}
F_{I} & =\sum_{s=1}^{s=N_{s}} B^{(s)} A^{(s)^{+}} B^{(s)^{T}} ; \\
G_{I} & =\left[\begin{array}{lll}
B^{(1)} R^{(1)} & \ldots & B^{\left(N_{s}\right)} R^{\left(N_{s}\right)}
\end{array}\right] ; \alpha=\left[\begin{array}{lll}
\alpha^{(1)^{T}} & \ldots & \alpha^{\left(N_{s}\right)^{T}}
\end{array}\right]^{T} \\
e & =\left[\begin{array}{lll}
b^{(1)^{T}} R^{(1)} & \ldots & b^{\left(N_{s}\right)^{T}} R^{\left(N_{s}\right)}
\end{array}\right]^{T}
\end{aligned}
$$


Splitting the Lagrange multipliers as

$$
\begin{aligned}
\lambda= & \lambda_{0}+P \bar{\lambda} \\
\text { where } \quad & \lambda_{0}=G_{I}\left(G_{I}^{T} G_{I}\right)^{-1} e \\
& P=I-G_{I}\left(G_{I}^{T} G_{I}\right)^{-1} G_{I}^{T}
\end{aligned}
$$

the interface problem (8) is transformed into the semi-definite system

$$
\begin{aligned}
& \left(P^{T} F_{I} P\right) \bar{\lambda}=P^{T}\left(d-F_{I} \lambda_{0}\right) \\
& \alpha=\left(G_{I}^{T} G_{I}\right)^{-1} G_{I}^{T}\left(d-F_{I} \lambda\right)
\end{aligned}
$$

Hence, a solution of the original indefinite system of interface equations (8) can be obtained by applying a Preconditioned Conjugate Gradient (PCG) algorithm to the symmetric semi-definite interface problem (13). Such a procedure can also be viewed as a Preconditioned Conjugate Projected Gradient (PCPG) algorithm [3].

At every PCPG iteration, the projection steps require the solution of a coarse grid problem associated with the subdomain floating modes of the form

$$
\left(G_{I}^{T} G_{I}\right) \alpha=G_{I}^{T} w
$$

These coarse grid problems are solved by second level Conjugate Gradient iterations with a projection and re-orthogonalization technique $[\mathbf{2}, \mathbf{3}]$ in order to re-use the Krylov spaces computed at previous iterations.

2.2. FETI applied to semi-definite problems. When problem (1) is positive semi-definite, the null space directions $u_{i}$ verifying (2) also satisfies the subdomain-wise null space condition

$$
\begin{aligned}
A^{(s)} u_{i}^{(s)} & =0 \\
\sum_{s=1}^{N_{s}} B^{(s)} u_{i}^{(s)} & =0
\end{aligned}
$$

stating that the null space of the global problem is also a null space for the subdomains and satisfies the interface compatibility. Hence, at the subdomain level, the global null space vectors are linear combinations of the local floating modes, i.e

$$
u_{i}^{(s)}=R^{(s)} \theta_{i}^{(s)}
$$

and computing the null space for the global problem is equivalent to finding the amplitudes of the local null space directions such that

$$
\sum_{s=1}^{N_{s}} B^{(s)} R^{(s)} \theta_{i}^{(s)}=G_{I} \theta_{i}=0
$$

where $\theta_{i}=\left[\begin{array}{lll}\theta_{i}^{(1)^{T}} & \ldots & \theta_{i}^{\left(N_{s}\right)^{T}}\end{array}\right]^{T}$. Therefore, when $m$ null space vectors $u_{i}$ exist, $G_{I}$ is no longer full rank and the coarse grid operator $\left(G_{I}^{T} G_{I}\right)$ has a null space of dimension $m$ such that

$$
\left(G_{I}^{T} G_{I}\right) \theta_{i}=0 \quad i=1, \ldots m
$$

In this case, a solution exists for the dual interface problem (8) only if $e$ is in the range of $G_{I}$, i.e.

$$
\theta_{i}^{T} e=0 \quad i=1, \ldots m
$$


Expanding this condition further by using definition (9) yields

$$
\sum_{s=1}^{N_{s}} \theta_{i}^{(s)^{T}} R^{(s)^{T}} b^{(s)}=\sum_{s=1}^{N_{s}} u_{i}^{(s)^{T}} b^{(s)}=u_{i}^{T} b=0 \quad i=1, \ldots m
$$

Thus, a solution exists for the dual interface problem if $b$ is in the range of $A$ in the initial problem (1) and in that case a starting value $\lambda_{0}$ can be computed by (11). A solution to the coarse problem (15) can be found by building a generalized inverse $\left(G_{I}^{T} G_{I}\right)^{+}$if a direct solver is used. For distributed memory machines, an iterative algorithm is usually preferred: a non preconditioned Conjugate Gradient scheme can still be applied since the successive directions of descent remain in the range of $\left(G_{I}^{T} G_{I}\right)$.

However, it is important that the singularity of the coarse grid be detected in the FETI method and that the null space be computed in order to check for condition (21), otherwise the FETI iterations could proceed without converging.

\section{Conjugate Gradient iterations for semi-definite problems}

The method for checking the singularity of a symmetric semi-definite positive operator and for computing the associated null space consists in applying a Conjugate Gradient iteration scheme to

$$
A x=A y
$$

where $A y \neq 0$.

If the initial guess for $x$ is set to zero, the computed $x$ will be in the range of $A$ since the directions of descent of the Conjugate Gradient iteration are combinations of the residual of (22). Therefore, if the solution $x$ at convergence is equal to $y$ independently on the choice of $y$, we can state that $A$ is non-singular. Otherwise, $u=x-y$ yields a null space vector. In the latter case, the iteration is restarted with a new $y$ direction orthogonal to the null space already extracted: $x-y$ is now searched for in a deflated space.

Using a projection and re-orthogonalization technique for solving problems with multiple right-hand sides [2], the algorithm can be summarized as in Table 1. In this algorithm, the directions of descent stored in $X$ are normalized such that $X^{T} W=I$, $W$ storing the results of $A X$. Note that the null space of $A$ is usually computed with a very high accuracy. Hence the stopping criterion for the Conjugate Gradient should be $\left\|r^{k}\right\|<\epsilon\|b\|$ with $\epsilon$ very small.

Clearly, two major issues remain to be cleared in algorithm 1 , namely how to set the vectors $y$ and what criterion to apply for the condition $x-y \neq 0$. Choosing $y$ correctly is of crucial importance for the success of the algorithm: on one hand it should not be in the null space so that $A y \neq 0$, and on the other hand, it should contain enough null space components so that $x-y=0$ occurs only if all the null space vectors have been extracted. It is clear that the choice of $y$ as well as the criterion for $x-y \neq 0$ should be based on an estimate of the condition number of $A$ which can be gathered from the Conjugate Gradient coefficients. Nevertheless, in the next section we propose a simple and efficient technique for choosing $y$ and checking for $x-y \neq 0$ when algorithm 1 is applied to the coarse grid problem 15 in FETI for structural problems. 
TABLE 1. Iterations for extracting the null space of $A$

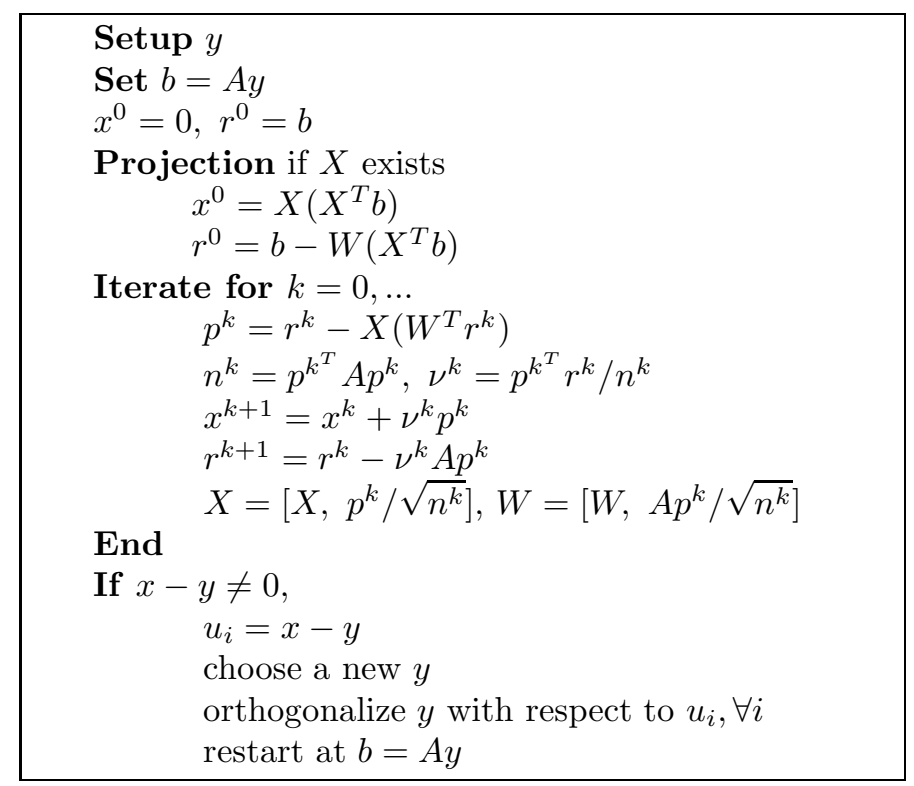

\section{Preliminary coarse grid iterations in FETI}

The computational cost of the procedure described in 1 can be significant since the number of descent directions for computing the entire null space with a good accuracy can be large. For instance, if applied to the iterative solution of the non decomposed problem (1), the singularity check would cost more than the actual computation of the solution.

As explained in section 2.2, the singularity issue for FETI appears in the coarse grid problem which dimension is very small compared to the dimension of $u$. Moreover, for the coarse grid problem, the complete Krylov space is needed anyway during the FETI iterations. Hence, solving a set of preliminary coarse grid problems of the form $\left(G_{I}^{T} G_{I}\right) x=\left(G_{I}^{T} G_{I}\right) y$ for finding the null space of $\left(G_{I}^{T} G_{I}\right)$ entails only a small overhead cost.

Note that for the primal Schur complement approach [6], algorithm 1 can be applied to the assembled Schur complement. This would however induce an unacceptable computational cost since it would amount to solving the entire interface problem several times. If however the balancing method version of the primal Schur method is used $[\mathbf{7}, \mathbf{6}]$, the singularity check can be performed at a low cost on the coarse grid operator associated to the Neumann preconditioner in a way similar to what is presented here for FETI.

Null vector criterion. To define a criterion for $x-y \neq 0$ in algorithm 1 , we decompose $y$ into

$$
y=\bar{y}+\sum_{i=1}^{m} \theta_{i} \beta_{i}
$$

where $\bar{y}$ is the component of $y$ in the range of $\left(G_{I}^{T} G_{I}\right)$. Since by construction the solution $x$ is in the range of $\left(G_{I}^{T} G_{I}\right), x-y \simeq \sum \theta_{i} \beta_{i}$. We then assume that the 
successive starting vectors $y$ are chosen to ensure a good representation of the null space, i.e. so that

$$
\left\|\sum_{i=1}^{m} \theta_{i} \beta_{i}\right\|>\|y\| / N \quad \text { if } m \neq 0
$$

when the dimension $N$ of the coarse grid is not trivially small. Hence we state that $x-y \neq 0$ if

$$
\|x-y\|>\|y\| / N
$$

Choosing the starting vectors. Let us remind that $G_{I}$ is the restriction of the local null spaces to the interface boundary and the columns of $R^{(s)}$ are usually orthonormalized so that they represent the rigid translational modes and the rotation modes around the nodal geometric centers (or around the center of gravity if the orthogonality is enforced with respect to the mass matrix). Since $G_{I} y$ represents the interface displacement jumps for local rigid body displacements of amplitude $y$, choosing

$$
y_{1}=\left[\operatorname{diag}\left(G_{I}^{T} G_{I}\right)\right]^{-1}\left[\begin{array}{ccccc}
1 & 1 & 1 & 1 & \ldots
\end{array}\right]^{T}
$$

all local translational and rotational modes around the local centers are included, and therefore the resulting displacement field is not compatible on the interface: $G_{I} y_{1} \neq 0$. The initial vector $y_{1}$ in $(26)$ is scaled by the diagonal of $\left(G_{I}^{T} G_{I}\right)$ in order to account for the fact that subdomains may have very different sizes thus different boundary displacements for the rotational modes.

Since $y_{1}$ is non-zero for all local rigid body modes, condition (24) is satisfied in practice. Based on similar mechanical considerations, the next initial vectors $y$ are then chosen as follows

$$
\begin{aligned}
& y_{2}=\left[\operatorname{diag}\left(G_{I}^{T} G_{I}\right)\right]^{-1}\left[\begin{array}{lllll}
1 & 0 & 1 & 0 & \ldots
\end{array}\right]^{T} \\
& y_{3}=\left[\operatorname{diag}\left(G_{I}^{T} G_{I}\right)\right]^{-1}\left[\begin{array}{lllll}
1 & 0 & 0 & 1 & \ldots
\end{array}\right]^{T} \quad \ldots
\end{aligned}
$$

The technique proposed in this paper for the FETI method has been implemented in the finite element analysis code SAMCEF and several free-free structures have been analyzed using this technique. In the next section we describe some of the test results.

\section{Application examples}

Free-free plane stress example. To illustrate the robustness of the proposed technique, let us first consider a square plane stress structural model decomposed into $8 \times 8$ square subdomains, each subdomain containing $10 \times 10$ finite elements (Fig. 1). No Dirichlet boundary conditions are applied to this two-dimensional problem so that every subdomain has 3 rigid body modes, the coarse grid problem is of dimension 192 and there are 3 global rigid body modes $(m=3)$.

Applying the iteration scheme 1 to the coarse grid operator $\left(G_{I}^{T} G_{I}\right)$ with a tolerance for the Conjugate Gradient iterations of $\epsilon=10^{-14}$ yields the correct three global rigid body modes. The convergence of the scheme for extracting the successive $\theta_{i}$ is described in Fig. 1. The convergence curves show that the number of iterations decrease every time a new null space component is searched for due to the projection and re-orthogonalization steps. Let us remind the reader that the directions computed and stored in this pre-processing step are used later on when the 


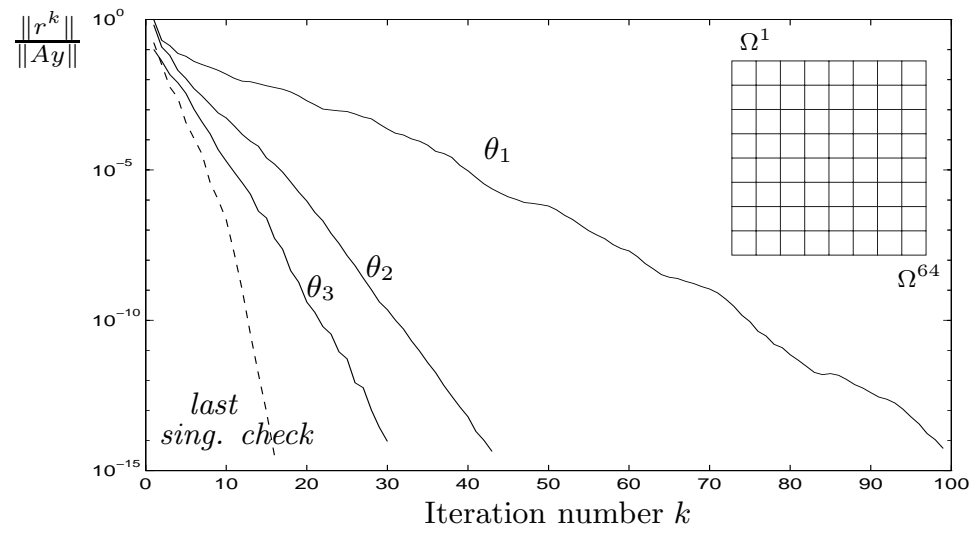

Figure 1. A free-free plane stress example

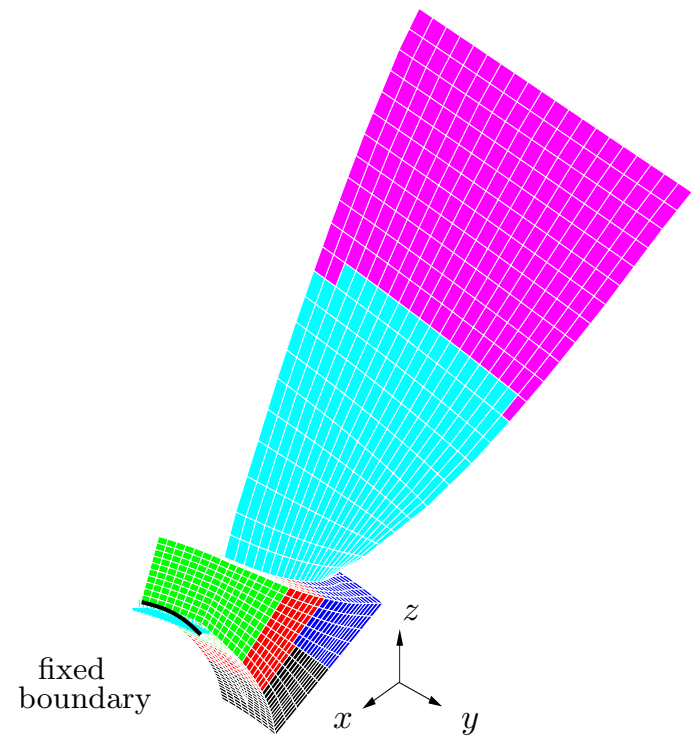

Figure 2. Decomposition of a blade model with 3 rigid body modes

linear system is actually solved by applying a projection and re-orthogonalization technique.

Analysis of a blade. We now present an example representing a realistic turbine blade modeled by brick elements. The model is decomposed into 6 subdomains and the displacements are fixed in the vertical direction on a curved edge at the base of the blade (Fig. 2). Note that because the fixed boundary is curved and belongs to the $(z, y)$ plane, the vertical constraints not only restrain the vertical translation and the rotation about the $x$ axis, but they also restrain the rigid rotation about the $y$ axis. Hence only 3 global rigid body modes exist.

Since the fixed boundary is only slightly curved, the problem of detecting the local floating modes for the constrained substructure is badly conditioned. However 
this does not affect the conditioning of the coarse grid problem. Applying algorithm 1 , we found the exact 3 rigid body modes. Note that if the operator $A$ of the entire structure would have been factorized, detecting the global rigid body modes would have been much more difficult.

\section{Conclusion}

In this paper we have addressed the problem of checking the singularity of a problem and computing the associated null space within the iterative solution procedure of a linear system. The method has been adapted to the FETI method in structural mechanics. A simple, low cost and robust technique has been proposed. It requires only preliminary iterations on the coarse grid problem associated to the subdomain null spaces and uses existing FETI technology. Hence our method entails only small modifications to the FETI algorithm and minor computational costs. The effectiveness of the procedure was demonstrated on some relevant examples. Equipped with this important singularity check, the FETI method can be used as an efficient solver in general static and free vibration analysis.

\section{References}

1. Ansys powersolver, USACM-Net Digest, July 271995.

2. C. Farhat, L. Crivelli, and F.X. Roux, Extending substructures based iteratives solvers to multiple load and repeated analyses, Comput. Methods Appl. Mech. Engrg. 117 (1994), 195209.

3. C. Farhat and F. X. Roux, Implicit parallel processing in structural mechanics, Comput. Mech. Adv. 2 (1994), no. 1, 1-124, North-Holland.

4. M. Géradin, D. Coulon, and J.-P. Delsemme, Parallelization of the SAMCEF finite element software through domain decomposition and FETI algorithm, Internat. J. Supercomp. Appl. 11 (1997), 286:298.

5. M. Géradin and D. Rixen, Mechanical vibrations, theory and application to structural dynamics, 2d ed., Wiley \& Sons, Chichester, 1997.

6. P. LeTallec, Domain-decomposition methods in computational mechanics, Comput. Mech. Adv. 1 (1994), 121-220, North-Holland.

7. J. Mandel, Balancing domain decomposition, Comm. Numer. Methods Engrg. 9 (1993), 233241.

Department of Applied Science, LTAS, University of Liège, 4000 Liège, Belgium

Current address: Department of Aerospace Engineering Sciences, Center for Aerospace Structures, University of Colorado, Boulder CO 80309-0429

E-mail address: d.rixen@colorado.edu 